\title{
Protée
}

\section{La cinématographie de la figure géométrique}

\section{Hadassa Shannie}

Volume 27, numéro 1, 1999

La Mort de Molière et des autres

URI : https://id.erudit.org/iderudit/030542ar

DOI : https://doi.org/10.7202/030542ar

Aller au sommaire du numéro

Éditeur(s)

Département des arts et lettres - Université du Québec à Chicoutimi

ISSN

0300-3523 (imprimé)

1708-2307 (numérique)

Découvrir la revue

Citer cet article

Shannie, H. (1999). La cinématographie de la figure géométrique. Protée, 27(1), 57-62. https://doi.org/10.7202/030542ar

\section{Résumé de l'article}

Cet article s'arrête sur la façon dont Wilson, dans La Mort de Molière, combine différentes formes d'art tout en changeant leurs valeurs et logiques. Les figures géométriques sont retenues pour les fins de l'analyse. Dans sa vidéo, Wilson utilise en effet des figures géométriques selon les principes de la perspective utilisés en art. Il crée ainsi un amalgame de systèmes interprétatifs, les uns empruntés aux arts plastiques, les autres inhérents à son médium. Par la voie de ces figures géométriques, le vidéaste met en jeu un médium qui transgresse ses propres frontières. 


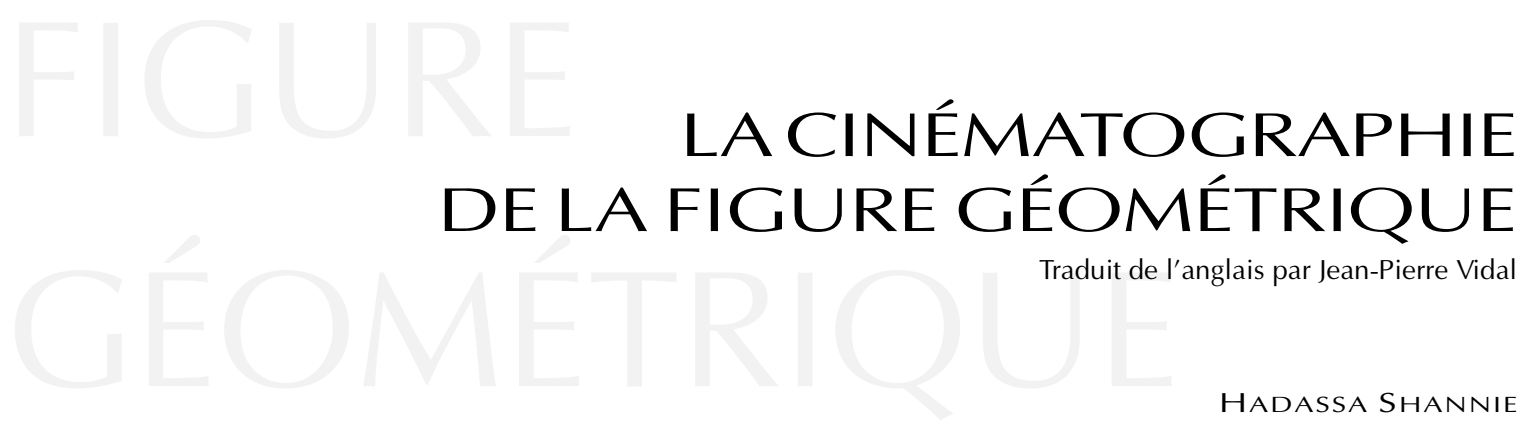

\section{INTRODUCTION}

Un des éléments majeurs de l'œuvre de Wilson est la relation entre diverses formes d'art comme la poésie, la peinture, le théâtre et le cinéma. Dans La Mort de Molière, chacune de ces formes d'art se trouve représentée au moyen de plusieurs types d'éléments. Le théâtre, par exemple, est représenté par Molière lui-même (en tant que sujet général de cette œuvre) ainsi que par la représentation de la scène, figurée par un carré blanc. Les arts plastiques le sont, entre autres, par la logique picturale de la figure géométrique.

La Mort de Molière n'est ni la première ni la plus cinématographique des vidéos de Wilson. Dans Stations (1982), par exemple, le sujet est le médium lui-même ${ }^{1}$. Une des questions soulevées par La Mort de Molière est aussi, une fois de plus, celle du médium; mais cette fois Wilson explore ce qui se passe quand le médium transgresse ses propres limites pour franchir celles d'un autre médium, allant ainsi encore plus loin que l'autoréférence. Cette transgression s'exprime par des déviations dans les fondements logiques de tel ou tel art spécifique, comme par exemple la peinture bidimensionnelle qui se met à bouger et l'image cinématographique, ordinairement constamment en mouvement, qui brusquement devient statique. Cette modification des fondements logiques provient de la tentative de Wilson de souligner la possibilité d'autonomie de chacun des divers arts en même temps que l'existence simultanée de tous les arts ${ }^{2}$. C'est que, dans La Mort de Molière, il franchit une étape supplémentaire: ne se satisfaisant plus de la réussite technique du montage des diverses formes d'art, il crée une série de contradictions entre les divers systèmes de sens ${ }^{3}$.

Le principal point que j'aimerais traiter ici, c'est que la conjonction d'éléments empruntés à diverses formes d'art, telle qu'on peut l'observer dans La Mort de Molière, se distingue par l'articulation de leurs systèmes de sens respectifs ${ }^{4}$; plus spécifiquement, l'acte créateur s'inscrit dans une forme d'art tandis que les autres fonctionnent dans le cadre de leurs propres principes explicatifs. La combinaison des diverses formes d'art s'effectue ainsi grâce à l'interchangeabilité de leurs principes explicatifs. J'ai choisi pour le montrer de concentrer mon analyse sur la figure géométrique et ses significations dans l'œuvre. 
La prédominance des formes graphiques est particulièrement évidente dans l'œuvre de Wilson. Il se sert de lignes droites et simples et ses combinaisons graphiques incluent une pléthore d'angles aux arêtes vives pour créer une sorte de mysticisme détaché, caractéristique de son esthétique ${ }^{5}$. L'usage qu'il fait des formes graphiques représente une pensée froide et mécanique, le type de formalisme qui domine dans son œuvre. La multiplicité des figures géométriques indique aussi un certain aplatissement de la troisième dimension. Dans sa sculpture environnementale Poles (1967), une œuvre très théâtrale dans laquelle Wilson travaille sur l'équilibre créé entre les lignes horizontales et les lignes verticales, une diagonale se forme à partir de l'inclinaison de l'œuvre, et cette diagonale apparaît comme une synthèse entre les forces horizontales et les forces verticales ${ }^{6}$. On peut ainsi voir, dans le même sens, la diagonale comme un symbole de l'approche esthétique de Wilson dans La Mort de Molière également: plutôt que l'harmonie de deux lignes parallèles, elle représente une existence partagée qui naît de la tension.

Dans La Mort de Molière, le sens général de la figure géométrique se développe pour donner une forme d'expression cinématique intéressante. Wilson se sert de ces figures pour créer un amalgame de systèmes interprétatifs, l'un emprunté aux arts plastiques, l'autre appartenant en propre au médium dans lequel il travaille. La contradiction qui en découle entre les deux systèmes de sens représente la principale caractéristique de la cinématographie de la figure géométrique dans cette œuvre et fait partie d'un exercice d'exploration du contexte de signification dont se sert Wilson pour sonder la nature de ce que nous appelons «l'art multimédia».

\section{LA FORMATION DES FIGURES GÉOMÉTRIQUES}

Dans la chambre à coucher de Molière, une pièce à angles droits, les visiteurs comme Galilée et Newton entourent son lit, lui aussi rectangulaire. La vidéo a été filmée dans un studio: une pièce carrée, divisée en plusieurs sections par la photographie d'un fauteuil à angles droits placée dans un cube. Un carré blanc représente la forme dominante du film. Des photographies de la pièce mettent en évidence le plancher, fait de carreaux gris à angle droit. La pièce a une fenêtre divisée elle aussi en carreaux.

Le film s'ouvre sur un plan du portrait de Molière, image rectangulaire transportée par sa fille. Comme le plancher et le fauteuil sont constamment filmés comme s'ils flottaient dans l'espace, un rectangle se forme à la base de l'image, mais il manque de définition. Les jeux d'ombre et de lumière produisent des marques longitudinales et transversales.

Une figure géométrique se trouve aussi produite par la façon dont sont placés les objets à l'intérieur du cadre et par les relations qui ainsi se créent entre eux, de même que par les lignes que «dessinent» sur l'écran les mouvements de la caméra: de brusques déplacements latéraux combinés à des soubresauts donnent l'illusion d'une mobilité verticale et horizontale qui vient zébrer l'image. Tous ces mouvements produisent une forme cinématographique bourrée d'angles droits, qui engendrent une expressivité froide et rigide.

\section{IMAGES FERMÉES, IMAGES OUVERTES}

L'image «fermée» appartient au style cinématographique dans lequel les détails du plan se trouvent délimités par une forme quelconque de frontière externe. $\mathrm{La}$ «fermeture" peut être créée par des objets - l'horizon dans la partie supérieure de l'image, des maisons, des arbres ou des groupes de gens sur les côtés -; elle peut même être produite par l'angle de prise de vue et par la lumière? ${ }^{7}$. Par contraste, «l'image ouverte» se trouve comme libérée de ses frontières géométriques, ses bords sont fluides, elle met l'accent sur la continuité et le plan-séquence. Le style cinématographique qui a recours à la fois à l'image fermée et à l'image ouverte représente, dans son essence, le transfert de la logique des arts plastiques au film.

En règle générale, Wilson ferme chaque plan. Il le fait au moyen d'objets, de personnes, de la lumière et des angles de prise de vue. En se concentrant sur l'image fermée, il lui donne un sens d'imperméabilité, 
comme si elle était scellée et impénétrable. Les délimitations d'images très explicites et rigides sont souvent le résultat de l'usage que fait Wilson de la figure géométrique comme instrument de cadrage, grâce à sa capacité d'introduire de l'ordre dans la segmentation. En outre, comme le style est celui de l'image fermée, on ne met pas l'accent sur la continuité ou le flux. Les relations entre les formes suffisent à l'indiquer: elles sont précises, froides, détachées, ne débordant jamais les unes sur les autres; tout au plus une forme peut-elle en inclure une autre (le cube contient un fauteuil, le plancher porte un carré), elles peuvent se rencontrer (les carrés du plancher, carrés noirs jouxtant un rectangle blanc section 5) ou se refléter l'une l'autre (la fenêtre se projette sur la pièce, un cube est réfléchi par l'autre). Tout est divisé en formes strictes.

Dans la cinématographie de La Mort de Molière, même si les figures sont des instruments de cadrage et que leur structure leur délimite un territoire bien défini, des bases et des fondations solides, certaines d'entre elles sont détachées - elles flottent dans l'espace (le carré blanc, par exemple; le plancher de la pièce, détaché de la base de l'image, laisse un espace indéfini au bas de cette même image). En d'autres mots, Wilson se sert des caractéristiques des figures géométriques tout en les niant en même temps.

L'attitude de Wilson à l'égard du style est semblable à son approche de la figure géométrique: l'affirmation s'y combine à la négation. Bien qu'il use en général du style image fermée, il fait aussi parfois appel au style image ouverte, comme le montrent ses efforts pour briser les limites et créer un type distinct de flux. Ces tentatives s'expriment avant tout dans sa volonté d'aller au-delà de la surface du plan, ou de se servir des mouvements erratiques de la caméra pour donner à l'espace un prolongement qui va plus loin que l'image individuelle (ceci est particulièrement évident dans les sections 1 et 10). Ses efforts pour faire éclater la surface bidimensionnelle de l'image se marquent dans la relation qui s'établit entre l'image du premier plan et une image supplémentaire, supposément placée en arrière-plan. Cette relation privilégiée, reflet de la tension entre les styles fermé et ouvert, va de pair avec l'extension de la profondeur de l'espace, qui dans cette œuvre acquiert une identité particulière.

\section{L'EXTENSION DE LA PROFONDEUR}

Dans cette vidéo, souvent l'espace tridimensionnel n'est pas représenté d'une façon propre au cinéma. Wilson suggère la profondeur au moyen de carrés superposés les uns aux autres, comme si l'on avait placé mécaniquement des figures géométriques les unes derrière les autres. Son approche génère une contradiction fondamentale entre les facteurs «bidimensionnels» et «tridimensionnels» mis en jeu dans cette œuvre.

Visuellement, une des fonctions les plus signifiantes de la figure géométrique consiste à créer l'illusion de la profondeur. Mais, dans La Mort de Molière, au lieu d'un paysage en trois dimensions, ou même à la place d'une image qui créerait l'illusion de la profondeur au moyen d'une structuration iconique en forme de cube, nous sommes en face de nombreuses surfaces faites de deux couches bidimensionnelles superposées et légèrement décalées l'une par rapport à l'autre. Les images du paysage vues par une fenêtre dans les sections 5 et 8 , par exemple, sont construites ainsi. Dans la section 5 , nous voyons Molière au lit et derrière ce lit un carré appartenant au dossier du fauteuil; derrière ce carré, il y a l'image de la fenêtre avec ses traverses et, derrière ces traverses, nous apercevons l'image d'un paysage immobile qui apparaît comme une surface bidimensionnelle. Ainsi, quatre plans spatiaux apparaissent placés l'un derrière l'autre. Une situation similaire se retrouve dans la section 8: Molière est dans son lit, une sœur est assise au pied du lit, une autre se tient debout de l'autre côté, tournant le dos à Molière; derrière elles, le mur de la chambre et, supposément derrière ce mur, la fenêtre avec ses traverses, qui semble «située» derrière le mur parce qu'elle forme un renfoncement par rapport au mur. L'ensemble de la composition paraît constitué de cinq plans spatiaux bidimensionnels. Bien que tout ceci soit très statique, nous percevons le 
mouvement d'un léger changement continu : la sœur debout bouge la première; quand elle quitte le cadre, deux femmes entrent; plus tard, c'est le mouvement des mains de Molière qui s'imposera à l'attention. Ces divers mouvements font contraste avec l'immobilité des autres éléments.

Ce qui contribue à l'impression de profondeur, ce sont les images et les objets eux-mêmes tout autant que leur position dans le cadre. C'est ainsi qu'on voit un rideau au premier plan d'une pièce, dans les sections 5 et 7 , ou que, dans la section 6, la sœur est filmée près d'un piano, en avant-plan, et deux chaises sont placées derrière le piano et la sœur. Cette combinaison d'images crée une composition formelle qui donne l'illusion de la profondeur, une profondeur dont les origines appartiennent aussi au système de sens des arts plastiques.

De plus, la façon dont l'espace de l'image se trouve ainsi ouvert ajoute une autre dimension encore: celle du système de sens du cinéma. La technique adoptée par Wilson combine l'extension statique de la profondeur spatiale à la création du mouvement dans le plan. D'un côté, l'image représente un espace qui s'étend le long d'un axe jusqu'aux extrémités de la profondeur du champ, mais cette expansion n'est pas dynamique, elle ne change pas, l'impression de profondeur vient des «superpositions de carrés». De l'autre, la caméra pénètre le cadre à de nombreuses reprises et fait éclater ses limites, étendant ainsi l'espace, dont la présence fait sens, généralement par des panoramiques qui balaient toute la largeur $\mathrm{du}$ cadre à la recherche, un peu futile, d'un objet quelconque (voir les sections 1, 4 et 10). Dans le second panoramique de la section 1, la caméra glisse sur des gens qui paraissent littéralement statufiés. Soudain, au milieu du panoramique, un personnage se détourne et quitte le cadre. La conjonction des mouvements de la caméra, de la stase artificielle des personnages et du mouvement soudain de l'un d'entre eux produit une grave contradiction entre les systèmes. La même chose se produit dans la section 10: le cadre est statique et les mouvements erratiques de la caméra interfèrent avec sa sérénité et créent un autre «carré» d'action à l'avant-plan. Ainsi, deux mises en scène contradictoires opèrent concurremment: une statique en arrière-plan et une dynamique en avant-plan. Dans la section 2, la caméra tressaute et effleure les visages de personnages assis sur des chaises; comme il n'y a pas alors de profondeur de champ, l'image qu'en reçoit le spectateur est celle de gens qui semblent «suspendus» au mur. Là encore, la tension entre les mouvements de la caméra au-dessus d'eux et la bidimensionnalité et l'inertie des cadrages qui délimitent les personnages se donne libre cours.

Ici Wilson souligne la contradiction entre deux systèmes de sens: celui des beaux-arts et celui du cinéma. L'image ne transmet pas une perception visuelle uniforme, mais instaure plutôt une tension entre les carrés de l'avant-plan et ceux de l'arrièreplan; entre l'image fermée et l'image ouverte. Il en résulte un message ambivalent ${ }^{8}$.

\section{L'AMALGAME DES SYSTĖMES DE SENS}

Dans bien des œuvres de Wilson, on peut détecter des moments isolés où se produit l'amalgame de deux systèmes de sens, c'est-à-dire une tentative pour œuvrer dans un médium selon les principes explicatifs d'un autre. Dans une conversation avec Umberto Eco, Wilson a déclaré qu'il croyait à la possibilité de faire une performance qui permette au public de quitter la salle pour aller prendre un café puis revenir, exactement comme les gens font dans une exposition: il s'agirait en fait d'une pièce qui n'aurait ni début ni milieu ni fin ${ }^{9}$. Une telle approche dénote une attitude qui refuse le système de sens du théâtre.

Bien qu'il y ait dans l'œuvre de Wilson de nombreux exemples de cette attitude, c'est dans $L a$ Mort de Molière qu'elle se change en véritable méthode, en exercice de construction d'un contexte de sens. Les solutions mises à jour dans La Mort de Molière reposent sur une tentative pour unifier les arts, dans une perspective idéologique plutôt que romantique; elles explorent ainsi la question de la possibilité d'une unification de plusieurs formes d'art en faisant intervenir leurs principes explicatifs ${ }^{10}$. La méthode utilisée consiste à altérer les fondements 
logiques du médium travaillé pour s'assurer que les principes explicatifs, qui appartiennent en propre à tel art spécifique, puissent fonctionner à l'extérieur de leur cadre habituel et à l'intérieur du champ d'une autre forme d'art.

Bien que Wilson soit passé maître dans de nombreuses formes d'art, son œuvre ne répond pas au concept d' "œuvre d'art totale»; au contraire, il refuse par certains côtés l'harmonie que pourrait peut-être engendrer la fusion de différentes formes d'art à l'intérieur d'une seule œuvre. Il préfère plutôt créer une tension entre des éléments qui, au départ, appartiennent à diverses formes d'art et interroger leur cohabitation.

En conclusion, on peut dire que Robert Wilson ne cherche pas à créer l'harmonie entre les arts. Il préfère mettre à jour les contradictions qui opposent leurs systèmes de sens. Pour ce faire, il fait appel à toutes les techniques disponibles d'une façon qui interdit leur synchronisation: la vidéo étend les dimensions de l'espace en appliquant les principes des beaux-arts; le théâtre, volontairement représenté par un de ses créateurs (Molière), comme s'il ne parvenait à faire naître ni personnages ni univers de fiction; la scène, qui n'est qu'une illusion produite par de la lumière dans un carré blanc; l'image, du moins celle de Molière (portée par sa fille au début de la vidéo), est ressuscitée en scène dramatique. Le résultat ressemble à une situation dans laquelle les impressions reçues par l'un de nos sens seraient en fait stimulées par d'autres. C'est dans des cas semblables que les formulations synesthésiques finissent par avoir un sens pour nous: «des couleurs criardes», une «lumière froide», un «son» flamboyant, etc. ${ }^{11}$. Dans le domaine des arts, la question de savoir si de tels amalgames ont un sens, ou sont même simplement possibles, reste ouverte.

\section{Notes}

1. Thomas Woodruff, un artiste qui a travaillé avec Wilson, parle de cette œuvre vidéo dans L. Shuyer, "Thomas Woodruff», dans Robert Wilson and his Collaborators, New York, Theatre Communications Group, 1989, p. 181-183.

2. Comme il le fait, par exemple, quand il introduit des «tableaux vivants" au théâtre.

3. J'utilise l'expression «système de sens" pour désigner l'ensemble des principes explicatifs qui appartiennent à une forme d'art spécifique et définissent sa logique propre dans sa spécificité par rapport à celle des autres formes d'art.

4. M. Vanden-Heuvel remarque chez Wilson un changement global d'approche, mais il le fait d'une toute autre perpective et ses remarques ne portent pas sur La Mort de Molière. Cf. Performing Drama/Dramatizing Performance, Michigan University Press, 1993, p. 165.

5. J. Birringer traite de cette question dans "Wilson/Wagner", Performing Arts Journal, n 43, janvier 1993, p. 64. Voir aussi Shuyer, 1989, p. 1985; Bigsby, A Critical Introduction to 20th Century American Drama-3, Cambridge, Cambridge University Press, 1982, p. 162.

6. R. Stearns, «Robert Wilson: From a Theater of Images », dans C. Nelson (sous la dir.), Robert Wilson: The Theater of Images, New York, Harper \& Row, 1980, p. 37.

7. Sur «l'image fermée» et "l'image ouverte», voir I. Avisar, Film Art: The Techniques and Poetics of Cinematic Expression (en hébreu), Tel-Aviv, The Open University, 1995, p. 117. Le style "image fermée" peut être observé, par exemple, dans certains films de Pasolini, des frères Taviani et souvent dans les films de Jean Renoir.

8. Une des choses importantes que l'on peut dire sur l'image visuelle de la figure géométrique construite par Wilson, c'est qu'elle est influencée par l'approche cubiste, mais je ne puis traiter de cette question ici.

9. Robert Wilson et Umberto Eco, «A Conversation », Performing Arts Journal, no 43, janvier 1993, p. 5 .

10. La description que propose Bonnie Marranca de cette union des arts, telle qu'on peut la voir à l'œuvre dans A Letter for Queen Victoria (1996), peut être qualifiée d'approche romantique: il s'agit d'unifier tous les arts dans une atmosphère spirituelle illusionniste et mystique. Cf. The Theatre of Images, Baltimore \& London, The Johns Hopkins University Press, 1996, p. 39.

11. E. H. Gombrich, Art $\mathcal{E}$ Illusion: a Study in the Psychology of Pictorial Representation, Washington, Phaïdon, 1959, p.310. 


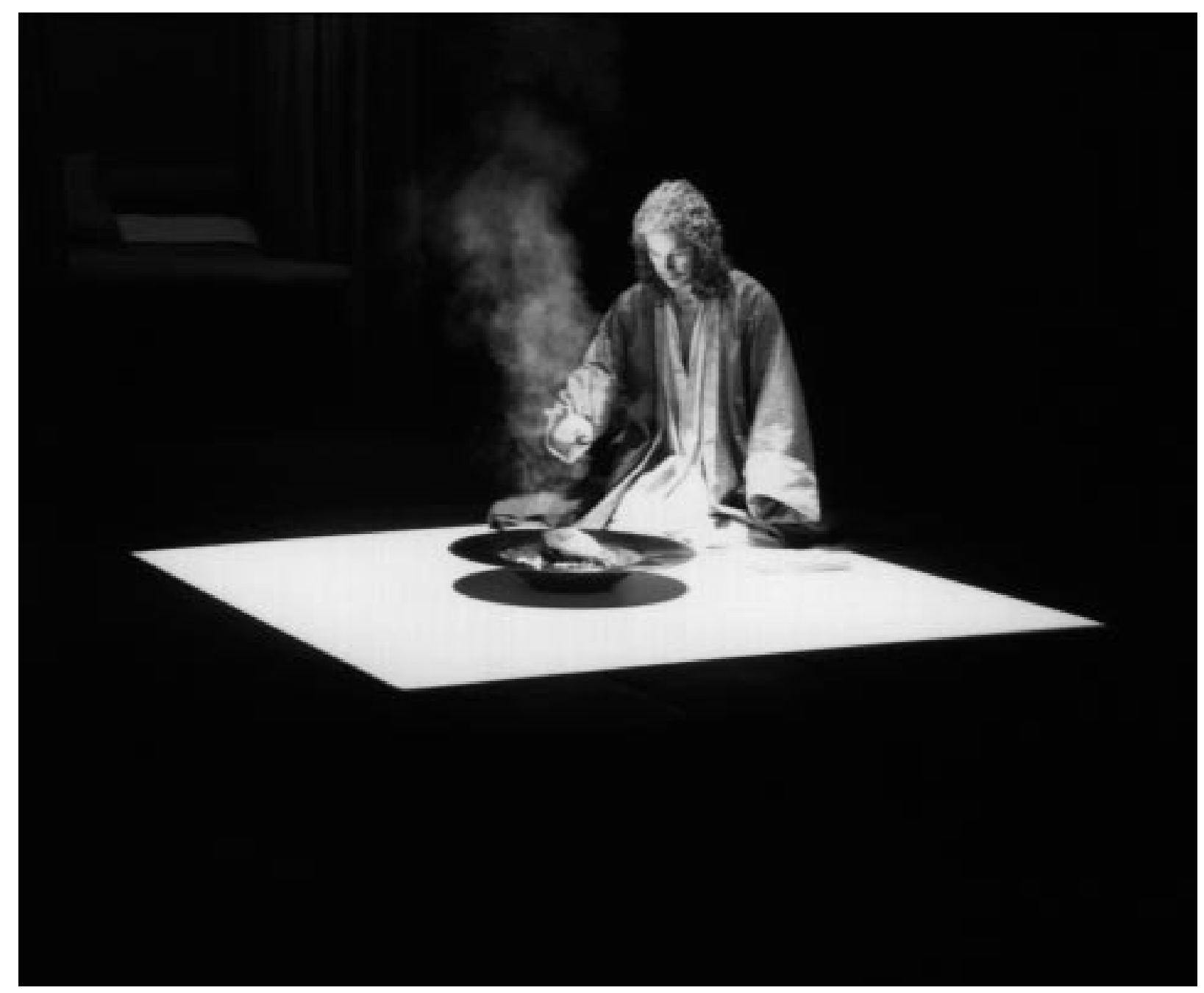

La Mort de Molière de Robert Wilson. Photo INA. 